HUB-EP-97/63

\title{
Critical exponents in U(1) lattice gauge theory with a monopole term *
}

\author{
G. Damm ${ }^{a}$, W. Kerler ${ }^{a, b}$ \\ ${ }^{a}$ Fachbereich Physik, Universität Marburg, D-35032 Marburg, Germany \\ b'Institut für Physik, Humboldt-Universität, D-10115 Berlin, Germany
}

We investigate critical properties of the phase transition in the four-dimensional compact U(1) lattice gauge theory supplemented by a monopole term for values of the monopole coupling $\lambda$ such that the transition is of second order. It has been previously shown that at $\lambda=0.9$ the critical exponent is already characteristic of a second-order transition and that it is different from the one of the Gaussian case. In the present study we perform a finite size analysis at $\lambda=1.1$ to get information wether the value of this exponent is universal.

\section{INTRODUCTION}

Our investigations are based on the Wilson action supplemented by a monopole term [1]

$S=\beta \sum_{\mu>\nu, x}\left(1-\cos \Theta_{\mu \nu, x}\right)+\lambda \sum_{\rho, x}\left|M_{\rho, x}\right|$

where $M_{\rho, x}=\epsilon_{\rho \sigma \mu \nu}\left(\bar{\Theta}_{\mu \nu, x+\sigma}-\bar{\Theta}_{\mu \nu, x}\right) / 4 \pi$ and the physical flux $\bar{\Theta}_{\mu \nu, x} \in[-\pi, \pi)$ is related to the plaquette angle $\Theta_{\mu \nu, x} \in(-4 \pi, 4 \pi)$ by $\Theta_{\mu \nu, x}=$ $\bar{\Theta}_{\mu \nu, x}+2 \pi n_{\mu \nu, x}$ [2]. We use periodic boundary conditions.

Studies of energy distributions with a newly developed dynamical algorithm have indicated that with increasing $\lambda$ the strength of the first order transition decreases such that the transition ultimately gets of second order [3,4]. Recently the behavior at larger $\lambda$ has been investigated in more detail [5]. The unexpected phenomenon has been revealed that the phase transition persists up to very large values of $\lambda$, where the transition moves to large negative $\beta$. Further it has been observed that (within errors) the monopole density becomes constant in the second-order region. By a finite size analysis it has been shown that at $\lambda=0.9$ the critical exponent is already characteristic of a second-order transition. Moreover, with the result $\nu=0.446(5)$ obtained, it turned out to be different from the exponent $\frac{1}{2}$ of the Gaussian

\footnotetext{
*Contribution to Lattice '97, International Symposium, Edinburgh, UK, 1997. This research was supported in part under DFG grant Ke 250/13-1.
}

case.

Similar results have been recently reported [6] for the Wilson action extended by a double charge term with coupling $\gamma$. There the first order transition, which occurs at $\gamma=0$, weakens with increasing $\gamma$ until it becomes of second order at a tricritical point. While for the usual periodic boundary conditions the second order region starts at negative $\gamma$, for a spherelike lattice it occurs already at $\gamma=0$ [6]. This could be due to inhomogeneities which tend to weaken the transition [8]. The result obtained in Ref. [6] for $\gamma=-0.2,-0.5$ is $\nu=0.365(8)$ which is smaller than the value mentioned above. Thus the two types of actions obviously lead to different results.

In the present study we continue the investigations with the action (1.1) because the addition of a monopole term is attractive in view of the close relation of monopoles to the phase structure. The increase of the finite-size effects with $\lambda$ observed in 5] has hinted at a possible increase of $\nu$. This suggests to check if for larger $\lambda$ the same exponent is obtained, which is done here by performing a finite size analysis at $\lambda=1.1$.

\section{DETERMINATION OF THE TRAN- SITION POINT}

In order to facilitate the determination of the transition point we have used the topological characterization of the phases [3.77. It is based on the fact that there is an infinite network of 

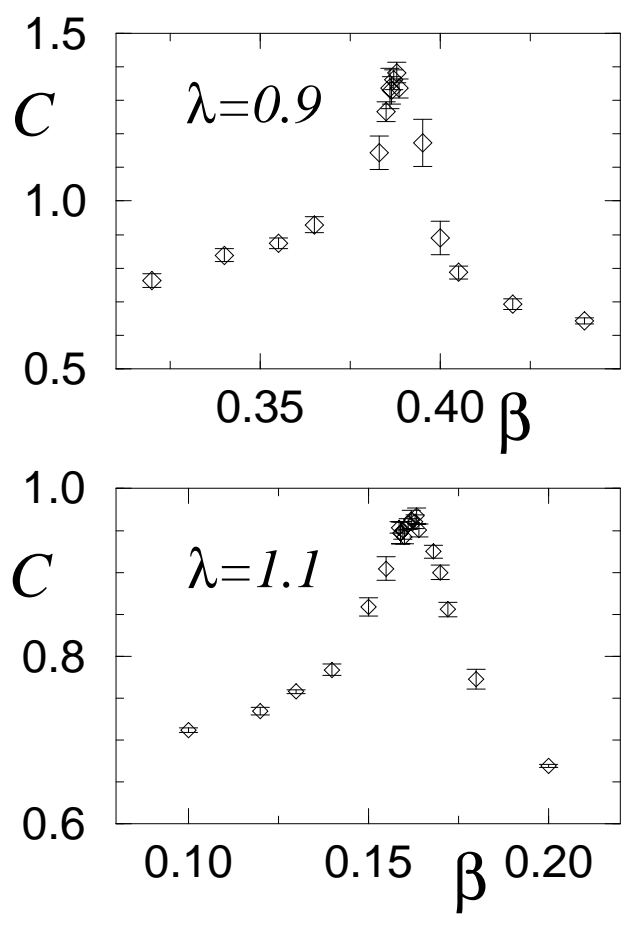

Figure 1. Specific heat $C$ as function of $\beta$ for $\lambda=0.9$ and 1.1 on the $8^{4}$ lattice.

monopole current lines in the confining phase and no such network in the Coulomb phase. On finite lattices "infinite" is to be defined in accordance with the boundary conditions [8]. For the periodic boundary conditions we are considering here, "infinite" is equivalent to "topologically nontrivial in all directions". While for loops the topological characterization is straightforward, to determine the topological properties of networks it is necessary to perform a more elaborate analysis based on homotopy preserving mappings [3, ]6].

Because the probability to find a network which is nontrivial in all directions takes values exactly 1 and 0 in the confining phase and in the Coulomb phase, respectively, it is a very advantageous order parameter. Indeed, with an infinite system a single configuration would be enough and on a finite lattice still few configurations are sufficient to discriminate between the phases.

Since on finite lattices different order parameters lead to slightly different critical $\beta$, to deter- mine the location of the maximum of the specific heat for larger $\lambda$ in an efficient way we first determine the critical $\beta$ from the topological order parameter and then find the maximum of the specific heat in an easy second step.

That finite size effects increase with $\lambda$ is indicated by the fact that the transition region becomes broader. From Figure 1 it can be seen that the width of the peak of the specific heat increases and that its height decreases with increasing $\lambda$.

\section{CRITICAL BEHAVIOR}

In order to obtain information on the nature of the phase transition we have investigated the finite-size scaling behavior of the maximum of the specific heat $C_{\max }$. It is expected to be

$C_{\max } \sim L^{d}$

if the phase transition is of first order and

$C_{\max } \sim L^{\frac{\alpha}{\nu}}$

if it is of second order, where $\alpha$ is the critical exponent of the specific heat and $\nu$ the critical exponent of the correlation length.

In Figure 2 we present results of simulations for $C_{\max }$ on lattices with $L=6,8,10,12$, for $\lambda=$ 0.9 obtained in Ref. [5] and for $\lambda=1.1$ obtained here, at the respective values of $\beta_{\mathrm{cr}}$. The fit to these data gives the values for $\frac{\alpha}{\nu}$ shown in Table 1. They are clearly quite far from 4 and thus the transition is not of first order.

Table 1

\begin{tabular}{llc}
\hline$\lambda$ & 0.9 & 1.1 \\
\hline$\underline{\alpha}$ & $0.485(35)$ & $0.289(68)$ \\
\hline$\nu$ & $0.446(5)$ & $0.466(8)$ \\
\hline$\beta_{c}(\infty)$ & $0.4059(5)$ & $0.1882(34)$ \\
$a$ & $-1.99(6)$ & $-2.29(10)$ \\
\hline
\end{tabular}

The results for $\nu$ listed in Table 1 are obtained using the hyperscaling relation $\alpha=2-d \nu$. They are different from the value $\frac{1}{2}$ of the Gaussian case. There is a slight increase with $\lambda$ which is beyond statistical errors. 


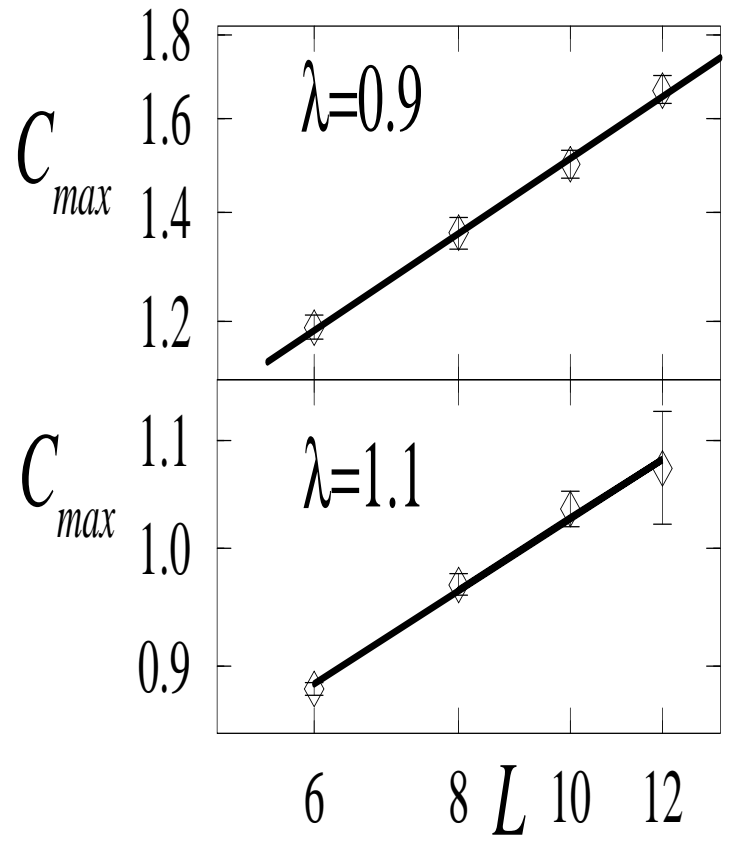

Figure 2. Maximum of specific heat $C_{\max }$ as function of lattice size $L$ for $\lambda=0.9$ and 1.1 at $\beta_{\text {cr }}$.

The critical $\beta$ is expected to behave as

$\beta_{\mathrm{cr}}(L)=\beta_{\mathrm{cr}}(\infty)+a L^{-\frac{1}{\nu}}$

From this relation, using the values of $\nu$ in Table 1 and our data for $\beta_{\mathrm{cr}}(L)$, the numbers given for $\beta_{\mathrm{cr}}(\infty)$ and $a$ in Table 1 are obtained.

\section{DISCUSSION}

The slight increase of $\nu$ with $\lambda$ observed could indicate a nonuniversal behavior or even hint at the possibilty that $\nu$ ultimately reaches the Gaussian value. However, finite size analyses on larger lattices and for still larger $\lambda$ appear necessary to decide these questions.

Different values for $\nu$ here and in Ref. [6] could signal different universality classes for the respective actions. One should, however, also be aware of the possibility of obtaining different limits when approaching from different regions of coupling space [9]. Further the use of different operators can lead to different values. Indeed, in an investigation of gauge balls with the double charge action for $\gamma=-0.2$ the values $\nu \simeq 0.35$ and 0.5 have been reported 10 .

It is interesting that recently theoretical arguments have been presented [11] predicting the set of exponents $1 / 3,5 / 11$ and $1 / 2$, which within errors are in agreement with the values observed in the simulations in Refs. [5,6, 10] and here.

With the non-Gaussian exponents continuum limits of four-dimensional pure $\mathrm{U}(1)$ gauge theory, nontrivial and not asymptotically free, appears possible. They would be different from the usual expectations for QED and would not be seen in perturbative approaches. Possibly then monopoles survive in the limit.

\section{ACKNOWLEDGMENT}

One of us (W.K.) wishes to thank M. MüllerPreussker and his group for their kind hospitality.

\section{REFERENCES}

1. J.S. Barber and R.E. Shrock, Nucl. Phys. B 257 (1985) 515.

2. T. DeGrand and D. Toussaint, Phys. Rev. D 22 (1980) 2478.

3. W. Kerler, C. Rebbi and A. Weber, Phys. Rev. D 50 (1994) 6984.

4. W. Kerler, C. Rebbi and A. Weber, Nucl. Phys. B 450 (1995) 452.

5. W. Kerler, C. Rebbi and A. Weber, Phys. Lett. B 392 (1997) 438.

6. J. Jersák, C.B. Lang and T. Neuhaus, Phys. Rev. Lett. 77 (1996) 1933; Phys. Rev. D 54 (1996) 6909.

7. W. Kerler, C. Rebbi and A. Weber, Phys. Lett. B 348 (1995) 565.

8. W. Kerler, C. Rebbi and A. Weber, Phys. Lett. B 380 (1996) 346.

9. M. Creutz, Phys. Rev. D 23 (1981) 1815.

10. J. Cox, W. Franzki, J. Jersák, C.B. Lang, T. Neuhaus and P.W. Stephenson, Nucl. Phys. B 499 (1997) 371.

11. J. Ambjørn, D. Espriu and N. Sasakura, preprint NBI-HE-97-22, hep-lat/9707095. 